\title{
Numerical Investigation of Confinement Losses in Semiconductor-core Optical Fibers
}

\author{
Mustafa W. Syed ${ }^{1}$, Ahmet E. Akosman ${ }^{2}$, Mustafa Ordu ${ }^{3}$ \\ ${ }^{I}$ Department of Physics, Bilkent University, Cankaya, Ankara 06800, Turkey. \\ ${ }^{2}$ School of Engineering, Computing and Construction Management, Roger Williams University, Bristol, RI 02809, USA. \\ ${ }^{3}$ UNAM - Institute of Materials Science and Nanotechnology, Bilkent University, Cankaya, Ankara 06800, Turkey. \\ waqar.syed@ug.bilkent.edu.tr,ordu@unam.bilkent.edu.tr
}

\begin{abstract}
The sources of the confinement losses in semiconductor-core optical fibers are numerically investigated by introducing fabrication-inspired imperfections such as micro-cracks and core ellipticity. The imperfections increase the losses up to $200 \mathrm{~dB} / \mathrm{m}$ at the targeted spectrum that is comparable to experimental findings.
\end{abstract}

Keywords-semiconductor-core fibers, confinement losses, mid-infrared transmission

\section{INTRODUCTION}

Low-loss mid-infrared (mid-IR) optical transmission has been demanded for a variety of applications including biomedical surgery, chemical sensing and defense systems [1]. While conventional silica core fibers provide a relatively low-loss window for near-IR transmission, the losses become considerable in the mid-IR region $(2-15 \mu \mathrm{m})$, leading to the need for alternative materials for mid-IR such as fluorides, chalcogenides and halide crystals [2-4]. Although such materials provide low transmission losses for the targeted mid-IR region, mechanical and chemical challenges such as devitrification at room temperature, water absorption and photodarkening under visible light limit their practical usage. Instead, semiconductors have been proven to be promising, featuring several superiorities such as low loss transmission windows in the IR spectrum, strong nonlinear properties, and stable chemical and physical properties [5]. Experimental studies show that the transmission losses are in the range of $0.9-30 \mathrm{~dB} / \mathrm{cm}$ in the mid-IR region [6-9]. Numerical modelling without taking the experimental challenges into account reveals these losses are two orders of magnitude lower, making such models an incomplete comparison for the experimental work. Fabrication methods of such fibers including the rod-in-tube method and high-pressure chemical vapor deposition (HP-CVD), can often lead to defects in the final structure of the optical fibers including micro-cracks on the core surface, elliptical cores, as well as core-cladding interface irregularities. These experimental setbacks are major sources of undesired attenuation that remain unaccounted in numerical models, drifting apart the numerical and experimental results.

As an effort to understand the loss mechanism, we propose realistic fabrication imperfections in the numerical models in the form of micro-cracks and core ellipticity. Various semiconductor-core silica glass-cladded step-index fibers including $\mathrm{Ge}$-core, $\mathrm{Si}$-core, $\mathrm{SiGe}$ alloy-core and $\mathrm{ZnSe}$ alloy cores were simulated using COMSOL Multiphysics in the wavelength range of 2-7 $\mu \mathrm{m}$, which is the transmission window for the studied materials. a)

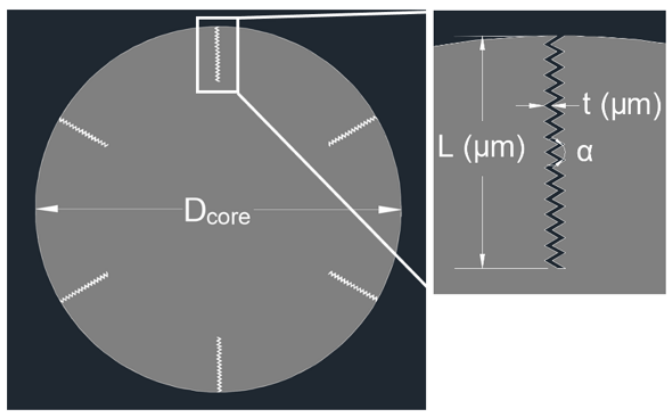

b)

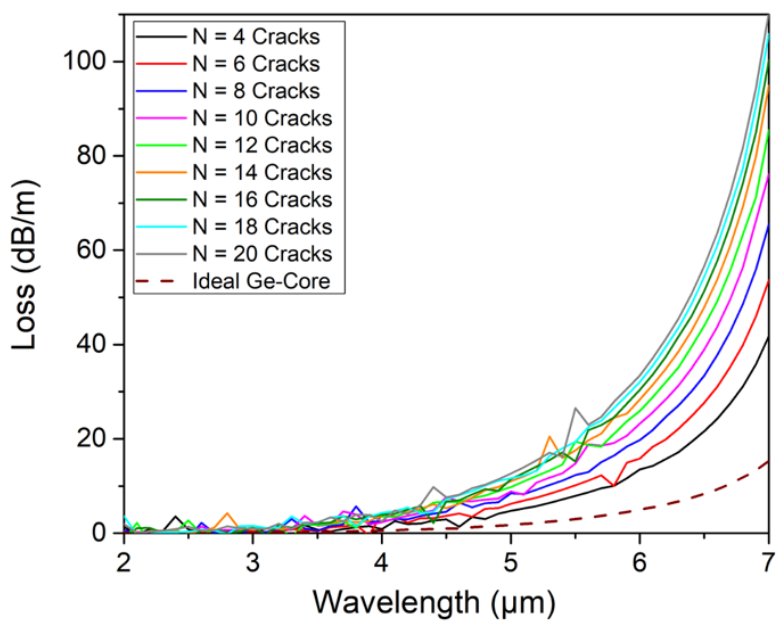

Fig. 1. a) Core cross-section of the modeled fiber with defects. The light gray region with the defects represents the semiconductor core whereas the darker outer part is the glass cladding. The micro-cracks consists of primary design parameters such as vertex angle $(\alpha)$, thickness (t) and length (L). b) Confinement loss of the Ge-core fiber with different number of cracks.

\section{NUMERICAL MODELLING OF FIBER DEFECTS}

We begin the modelling of the fibers by choosing the core diameter $\left(\mathrm{D}_{\text {core }}\right)$, cladding diameter $\left(\mathrm{D}_{\text {cladding }}\right)$ and crack length (L) as $10 \mu \mathrm{m}, 50 \mu \mathrm{m}$ and $1 \mu \mathrm{m}$, respectively. The crosssection of the fiber with the defects (cracks) is shown in Figure 1(a). The cracks were modeled based on several parameters: vertex angle $(\alpha)$, crack thickness $(t)$, number of cracks $(N)$ and ellipticity $(e) . \alpha$ was swept between $\pi / 6$ and $2 \pi / 3$, and $t$ was swept from $25 \mathrm{~nm}$ to $100 \mathrm{~nm}$. The optimal values of $\alpha$ and $t$ were calculated as $\pi / 3$ and $50 \mathrm{~nm}$, respectively. The ellipticity of the core was characterized by varying the ratio of the minor $(b)$ to major $(a)$ axis, and the ellipticity was calculated by $e=1-(\mathrm{b} / \mathrm{a})$. We found that successive increase in the ellipticity resulted in higher losses. However, we implemented $e$ as 0.10 , so as to make the fiber core a more realistic interpretation of the experimental findings. Then, we investigated the relationship between the confinement loss and the number of cracks, $N$. Varying $N$ 
imposed a significant impact on the loss profile as seen in Figure 1(b) with the Ge-core fiber. Losses increase with the number of cracks but the model becomes more complicated that limits the core region. Therefore, $\mathrm{N}$ was selected as 10 for the optimum modelling. While modelling the cracks, we had ensured that the field distribution of the light propagating through the fiber core was undisturbed by the presence of the cracks, leading us to consider minimum crack lengths. Figure 2(a) shows the effect of crack length, $L$, on the confinement loss of Ge-core fibers by sweeping $L$ between $1.0 \mu \mathrm{m}$ and 2.0 $\mu \mathrm{m}$. Although increasing crack lengths resulted in higher losses, we implemented $1.0-\mu \mathrm{m}$ long cracks, in order to ensure that the light was guided by the fiber without significant impact on the field distribution. Moreover, an irregular core-cladding interface was applied to our model by introducing multiple $\sim 100 \mathrm{~nm}$ long indents that can occur during the fiber drawing [5]. However the effect of the interface irregularity to the transmission was insignificant due to the presence of micro-cracks. Therefore, we excluded them from the models.

a)

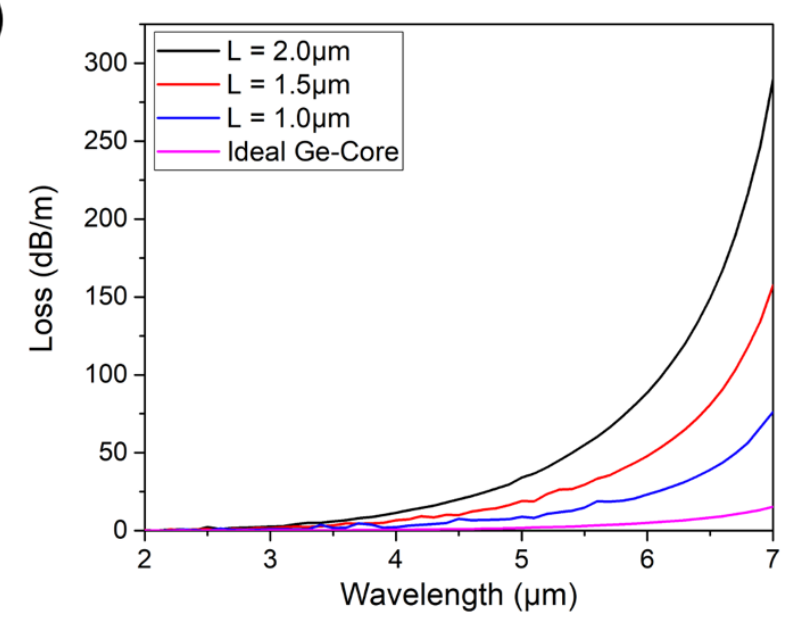

b)

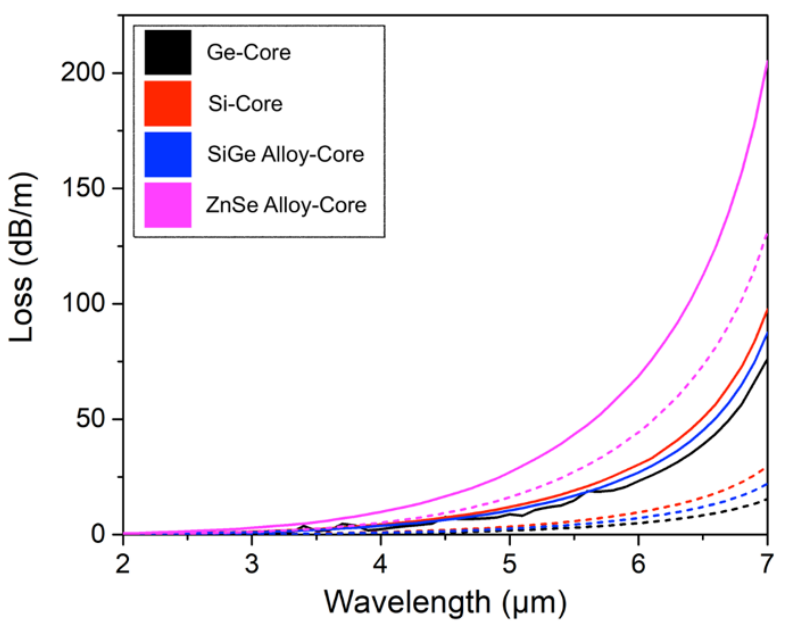

Fig. 2. a) Effect of the crack length on the transmission of the Ge-core fibers. b) Loss comparison of fibers with different core materials. The dotted and the solid lines represent ideal cores and the cores with the defect models, respectively.

\section{THE LOSSES OF SEMICONDUCTOR-CORE FIBERS}

The developed model was implemented to four different semiconductor core fibers: Ge, Si, SiGe alloy (50 at $\%$ and 50 at $\%$ ) and $\mathrm{ZnSe}$ alloy (50 at $\%$ and $50 \mathrm{at} \%$ ). All fibers have the same silica glass claddings for a better comparison. The parameters are selected as $\alpha=\pi / 3, t=50 \mathrm{~nm}, e=0.90, N=$ 10 cracks, and $L=1.0 \mu \mathrm{m}$. Figure 2(b) shows the confinement losses of the semiconductor-core fibers with the defected and ideal core models. At $6 \mu \mathrm{m}$, the losses were increased by 367 $\%, 216 \%, 275 \%$, and $55 \%$ for $\mathrm{Ge}, \mathrm{Si}, \mathrm{SiGe}$ and $\mathrm{ZnSe}$, respectively. In the case of Ge-core glass-cladded optical fibers, for instance, the numerical loss was increased from the original range of $0.03-29 \mathrm{~dB} / \mathrm{m}$ to $0.14-76 \mathrm{~dB} / \mathrm{m}$, thereby bridging the gap between the experimental and the numerical findings. To the best of our knowledge, this is the first study which focuses on incorporating experimental setbacks in numerical models. On the other hand, other loss mechanisms such as impurities and coupling losses could further fill the gap between the calculated losses and experimental values.

\section{CONCLUSION}

In this work, we have presented fiber models to reduce the numerical and experimental disagreement of confinement losses in semiconductor-core glass-cladded optical fibers by introducing fabrication related defects into the models, namely surface micro-cracks and core ellipticity. The proposed models improve the reliability of numerical findings and our understanding on the loss mechanism of the semiconductor-core fibers. Future efforts will focus on the losses related to the impurity diffusion between the corecladding regions.

\section{ACKNOWLEDGMENT}

This work received no external funding.

\section{REFERENCES}

[1] J. A. Harrington, "Infrared Fibers and Their Applications." SPIEInternational Society for Optical Engineering, (2003).

[2] J. S. Sanghera, et al., "Development and infrared applications of chalcogenide glass optical fibers," Fiber Integr. Opt. 19(3), 251-274, (2000).

[3] A. Sa'Ar, et al. "Optical and mechanical properties of silver halide fibers." OE/Fibers' 87. International Society for Optics and Photonics, (1987).

[4] S. Mitachi and T. Manabe. "Fluoride glass fiber for infrared transmission." Japanese Journal of Applied Physics 19.6: L313, (1980).

[5] M. Ordu and S. N. Basu. "Recent progress in germanium-core optical fibers for mid-infrared optics." Infrared Physics \& Technology 111: 103507, (2020).

[6] P. Mehta, et al. "Mid-infrared transmission properties of amorphous germanium optical fibers." Applied Physics Letters 97.7: 071117, (2010).

[7] J. Ballato, et al. "Silica-clad crystalline germanium core optical fibers." Optics Letters 36.5: 687-688, (2011).

[8] D. A. Coucheron, et al. "Laser recrystallization and inscription of compositional microstructures in crystalline SiGe-core fibres." Nature communications 7.1: 1-9, (2016).

[9] M. Ordu, et al. "Effect of thermal annealing on mid-infrared transmission in semiconductor alloy-core glass-cladded fibers." Advanced Fiber Materials 2.3: 178-184, (2020). 